\title{
Letter
}

\section{Perturbative evolution: a different approach at small $x$}

\author{
A. Donnachie ${ }^{1, a}$, P. V. Landshoff ${ }^{2, b}$ \\ ${ }^{1}$ School of Physics and Astromony, University of Manchester, Manchester, UK \\ ${ }^{2}$ DAMTP, Cambridge University, Cambridge, UK
}

Received: 2 June 2017 / Accepted: 14 July 2017 / Published online: 7 August 2017

(C) The Author(s) 2017. This article is an open access publication

\begin{abstract}
We propose an approach to DGLAP evolution at small $x$ that circumvents the usual problem that a perturbation expansion is not valid there. The data for the charm structure function are important to motivate the method, and it describes them much more successfully than the conventional approach.
\end{abstract}

\section{Introduction}

Although fixed-order perturbative QCD is widely applied to data for the proton structure function $F_{2}\left(x, Q^{2}\right)$, it is well known [1-6] that there are theoretical problems at small $x$. In this paper, we refine our previous approach [7] to overcoming this problem, which combines the DGLAP evolution equation with Regge theory.

An important motivation is provided by the HERA data [8] for charm electroproduction, which have $F_{2}^{c \bar{c}}\left(x, Q^{2}\right)$ behaving at small $x$ as the same fixed power $x^{-\epsilon_{0}}$, with $\epsilon_{0}$ close to 0.4 , down to very small $Q^{2}$. This is seen in Fig. 1 . We show only data having $y<0.55$, so that although strictly the data are for the reduced cross section, this differs little from $F_{2}^{c \bar{c}}\left(x, Q^{2}\right)$. The lines in Fig. 1 are the result of the calculations we describe in this paper of $F_{2}^{c \bar{c}}\left(x, Q^{2}\right)$ to nonleading order (NLO) in perturbative QCD. They are to be contrasted with calculations [9] in the conventional approach to perturbative evolution, two of which are shown in Fig. 2. The conventional approach ignores the need for resummation at small $x$, which we do not know how properly to do and which we circumvent. If anything, the photoproduction data in Fig. $1 \mathrm{~b}$ call for a rise even steeper than $\left(W^{2}\right)^{0.4}$, though the line in the figure does not include threshold effects, which will pull it down a little at the lower energies.

Partially supported by STFC grant ST/L000385/1.

a e-mail: Sandy.Donnachie@ manchester.ac.uk

be-mail:pvl@damtp.cam.ac.uk
Data for high energy $p p$ and $\bar{p} p$ elastic scattering and total cross sections have long been known to be dominated by socalled soft pomeron exchange, giving a power behaviour $s^{\epsilon_{1}}$ to the amplitude. First fits $[10,11]$ gave a value for $\epsilon_{1}$ close to 0.08 , but more recent data increase this $[12,13]$ to 0.096 . The emergence from HERA of data for $F_{2}\left(x, Q^{2}\right)$ revealed [14] that at small $x$ the power $x^{-\epsilon_{1}}$ alone did not fit and that a further term behaving as $x^{-\epsilon_{0}}$ needed to be added. The value of $\epsilon_{0}$ is about 0.4 , but with quite a large uncertainty, perhaps $10 \%$. For the complete $F_{2}\left(x, Q^{2}\right)$ both terms are needed to describe the data, but we have seen in Fig. 1 that for the charm component of $F_{2}\left(x, Q^{2}\right)$ the "hard-pomeron" term $x^{-\epsilon_{0}}$ is dominant, with extremely little room for the addition of the "soft-pomeron" term $x^{-\epsilon_{1}}$.

In leading order, perturbative QCD charm electroproduction is calculated from the gluon density $x g\left(x, Q^{2}\right)$, and to a very good approximation this is true also in nextto-leading order. Hence $\operatorname{xg}\left(x, Q^{2}\right)$ is dominated by hardpomeron exchange at small $x$-it behaves as $x^{-0.4}$ even at quite small $Q^{2}$ :

$x g\left(x, Q^{2}\right)=G\left(Q^{2}\right) x^{-\epsilon_{0}}$.

If we Mellin transform the quark and gluon densities by applying $\int_{0}^{1} \mathrm{~d} x x^{N-1}$, a power $u\left(Q^{2}\right) x^{-\epsilon}$ in the $u$-quark density, for example, becomes a pole $u\left(Q^{2}\right) /(N-\epsilon)$. Write

$$
\begin{aligned}
\mathbf{q}\left(Q^{2}\right)= & {\left[\begin{array}{c}
\Sigma\left(Q^{2}\right. \\
G\left(Q^{2}\right)
\end{array}\right] \Sigma\left(Q^{2}\right)=u\left(Q^{2}\right)+d\left(Q^{2}\right)+s\left(Q^{2}\right) } \\
& +c\left(Q^{2}\right)+\bar{u}\left(Q^{2}\right)+\bar{d}\left(Q^{2}\right)+\bar{s}\left(Q^{2}\right)+\bar{c}\left(Q^{2}\right),
\end{aligned}
$$

then

$Q^{2} \frac{\mathrm{d}}{\mathrm{d} Q^{2}} \mathbf{q}\left(Q^{2}\right)=\frac{\alpha_{S}\left(Q^{2}\right)}{2 \pi} \mathbf{P}\left(N=\epsilon, \alpha_{s}\left(Q^{2}\right)\right) \mathbf{q}\left(Q^{2}\right)$.

At nonleading order (NLO) the evolution matrix is

$\mathbf{P}\left(N, \alpha_{s}\right)=\mathbf{P}^{0}(N)+\frac{\alpha_{s}}{2 \pi} \mathbf{P}^{1}(N)$. 
Fig. 1 a Data [8] for charm electroproduction, with lines proportional to $x^{-0.4}$ and $\mathbf{b}$ for charm photoproduction compared with $\left(W^{2}\right)^{0.4}$

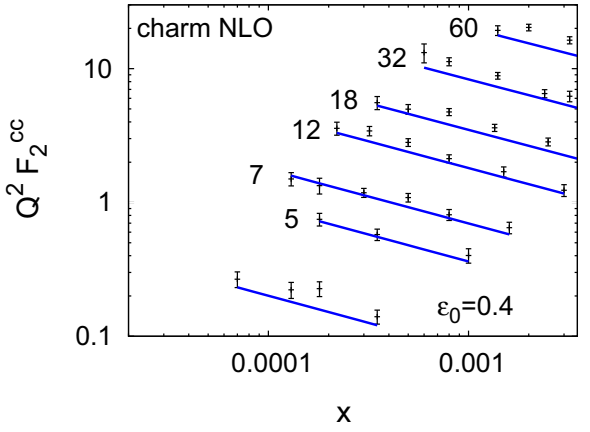

(a)

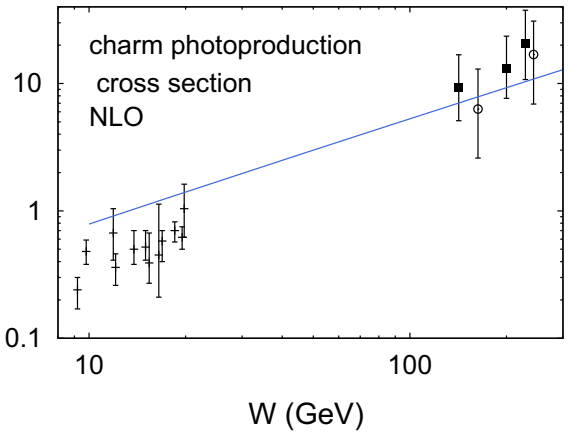

(b)
Fig. 2 Calculations [26] of $F_{2}^{c \bar{c}}\left(x, Q^{2}\right)$ at NLO and NNLO. In a the NNLO calculations are below the NLO, while in $\mathbf{b}$ they are above

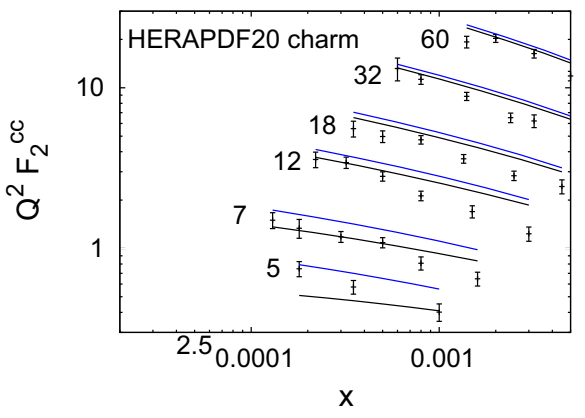

(a)

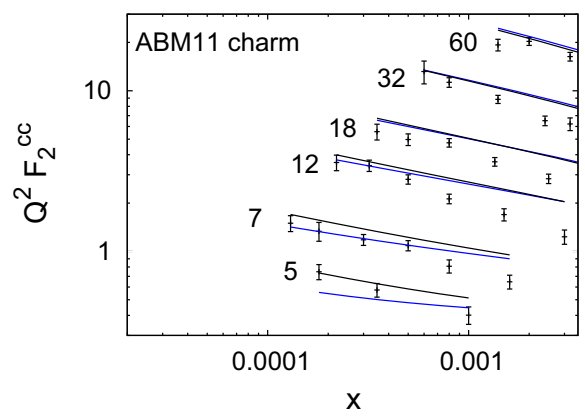

(b)
The problem with applying this to perturbative evolution at small $x$ lies in that, while the $q q$ and $q g$ elements of $\mathbf{P}^{0}(N)$ are finite at $N=0$, its other two elements, and those of $\mathbf{P}^{1}(N)$, have poles there [15]. This means that at $N=0.096$ the NLO evolution matrix is very different from its leading order (LO) version.

Of the four elements of the evolution matrix $\mathbf{P}(N)$, it is [15] $P_{q g}$ that differs most between LO and NLO. As is seen in Fig. 3a, for $N$ between 0.096 and $0.4 \operatorname{good}$ fits to $P_{q g}^{0}(N)$ and $P_{q g}^{1}(N)$ are

$P_{q g}^{0}(N)=2.59-2.21 N+1.085 N^{2}$,

$P_{q g}^{1}(N)=24.6 / N-28.2$.
As a model for the exact $P_{q g}(N)$ consider

$$
\begin{aligned}
P_{q g}= & P_{q g}^{0}(N)+C \sqrt{C^{2} N^{2}+N \alpha_{s} P_{q g}^{1}(N) / \pi}-C^{2} N \\
= & P_{q g}^{0}(N)+\alpha_{s} P_{q g}^{1}(N) /(2 \pi) \\
& -\alpha_{s}^{2}\left[P_{q g}^{1}(N)\right]^{2} /\left(8 \pi^{2} N C^{2}\right)+\cdots
\end{aligned}
$$

where $C$ is a constant. Figure $3 \mathrm{~b}$ shows the exact $P_{q g}$ in this model, together with the NLO and NNLO approximations to it. The conclusion from this model is that, provided the NLO and the NNLO approximations are not very different, then NLO is already a good approximation. Further, this is much more likely to be true for $N=0.4$ than for $N=0.096$.
Fig. 3 a The evolution matrix element [15] $P_{q g}$ for $\alpha_{s}=0.21$ at LO and NLO together with the fits (3a); $\mathbf{b}$ the exact $P_{q g}$ in the model (3b) (points) with the NLO and NNLO approximations

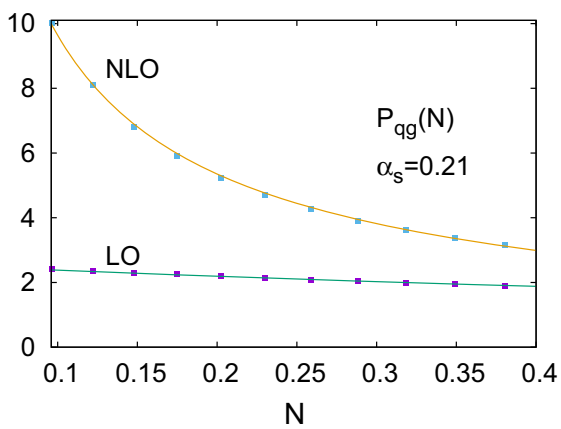

(a)

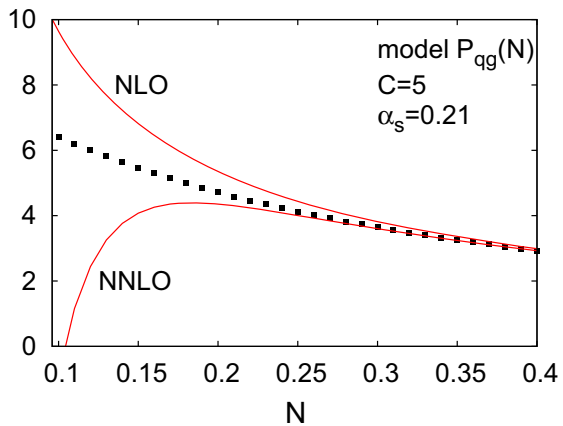

(b) 
Fig. 4 LO calculation of $F_{L}\left(x, Q^{2}\right)$ at the indicated values of $Q^{2}$ compared with data from ZEUS [16] and H1 [17]
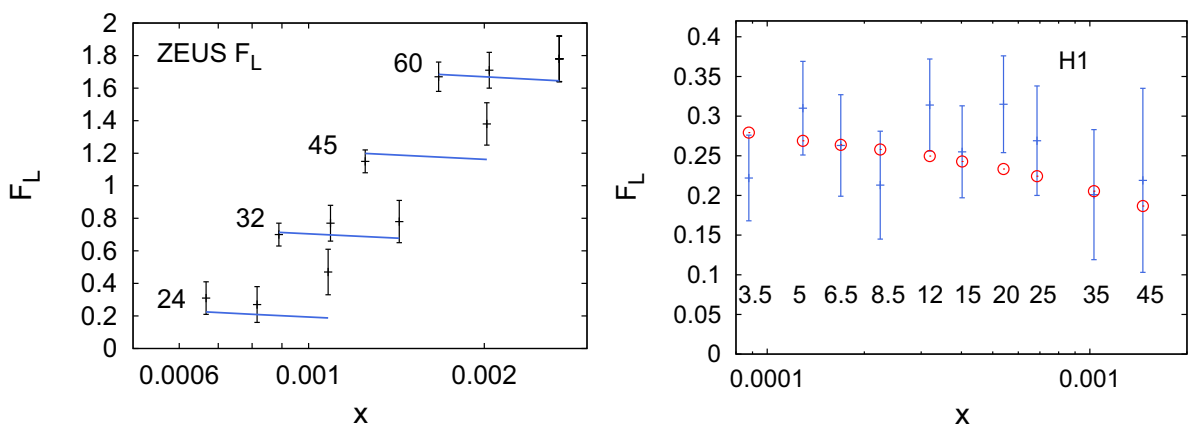

The problem with the perturbative expansion at small $x$ is implicit in all conventional applications of the DGLAP equation: for values of $Q^{2}$ for which the PDFs are fairly flat in $x$ at small $x$, that is the effective power $(1 / x)^{\epsilon}$ is small, it is not valid to use the DGLAP equation with $\mathbf{P}\left(N, \alpha_{s}\left(Q^{2}\right)\right)$ expanded in powers of $\alpha_{s}\left(Q^{2}\right)$.

But we have inferred that the contribution from soft pomeron exchange to the gluon density is negligibly small. So we apply NLO perturbative QCD evolution (2b) with $N=\epsilon_{0}$ to the coefficient of the hard-pomeron contribution to $F_{2}\left(x, Q^{2}\right)$. We have seen in Fig. 1 that the resulting gluon density leads to a highly successful calculation of $F_{2}^{c \bar{c}}\left(x, Q^{2}\right)$ to NLO. Figure 4 shows data $[16,17]$ that indicate that the same is true of a calculation to leading order (LO) of the longitudinal structure function $F_{L}\left(x, Q^{2}\right)$. Unlike for $F_{2}\left(x, Q^{2}\right)$, the NLO correction is known to be small [18].

\section{Fit to data for $F_{2}$}

The $\mathrm{H} 1$ and ZEUS experiments at HERA have agreed joint data for the proton structure function $F_{2}\left(x, Q^{2}\right)$. Their 2010 publication [19] both included results for the so-called reduced cross section

$\sigma^{\mathrm{red}}\left(x, y, Q^{2}\right)=F_{2}\left(x, Q^{2}\right)-\frac{y^{2}}{1+(1-y)^{2}} F_{L}\left(x, Q^{2}\right)$

and an extraction of $F_{2}\left(x, Q^{2}\right)$ from it, though only for events with $y$ less than about 0.5 . Their 2015 publication [20] gave results only for $\sigma^{\text {red }}\left(x, y, Q^{2}\right)$ and did not attempt to extract $F_{2}\left(x, Q^{2}\right)$.

We fit the small- $x$ 2010 HERA data [19] for $F_{2}\left(x, Q^{2}\right)$ to a sum of three powers of $x$ :

$$
F_{2}\left(x, Q^{2}\right)=A_{0}\left(Q^{2}\right) x^{-\epsilon_{0}}+A_{1}\left(Q^{2}\right) x^{-\epsilon_{1}}+A_{2}\left(Q^{2}\right) x^{-\epsilon_{2}} .
$$

Here, the first two terms are hard and soft pomeron exchange. The last term is reggeon exchange, that is, the families $f_{2}$ and $a_{2}$; its contribution for the small values of $x$ at which we work is small.
The only theoretical constraint we know on the three coefficient functions $A_{i}\left(Q^{2}\right)$ is that, as $Q^{2} \rightarrow 0, F_{2}\left(x, Q^{2}\right)$ vanishes linearly in $Q^{2}$ at fixed $2 v=Q^{2} / x$. We respect this constraint, even though our fit is for values of $Q^{2}$ some way from zero. This is sufficient for our purpose, because perturbative evolution is valid only for "large" values of $Q^{2}$. The theory does not tell how large but we shall find that $Q^{2}$ must be at least about $5 \mathrm{GeV}^{2}$.

We have tried various forms of the $A_{i}\left(Q^{2}\right)$. Several give much the same value of $\chi^{2}$ in the fit to the data, but rather different values of $\epsilon_{0}$, which is why there is still a rather large uncertainty in its value. In this paper we give the results for the choices

$$
\begin{aligned}
A_{i}\left(Q^{2}\right)= & X_{i}\left(Q^{2} /\left(Q_{i}^{2}+Q^{2}\right)\right)^{1+\epsilon_{i}}\left(1+2 Q^{2} / Q_{i}^{2}\right)^{0.15}, \\
& i=0,1,2 .
\end{aligned}
$$

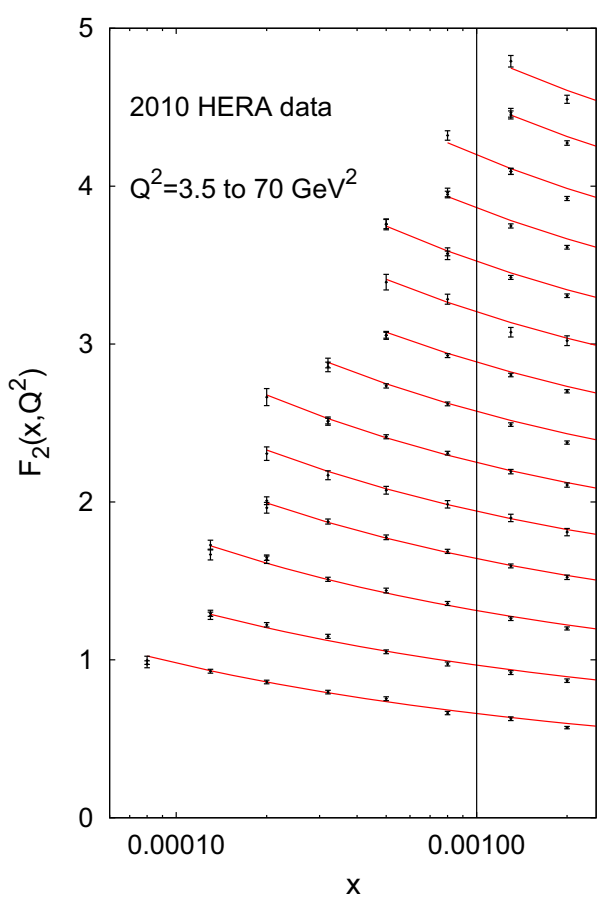

Fig. 5 fit to 2010 HERA data [19] - only data with $x<0.001$ are included in the fit 
We use the values

$\epsilon_{0}=0.4, \quad \epsilon_{1}=0.096, \quad \epsilon_{2}=-0.343$,

where the last two are extracted [23-25] from $p p$ and $\bar{p} p$ elastic scattering and total cross section data.

We confine attention to the small- $x$ data, which we take to mean $x<10^{-3}$. But we introduce into each term a power the case, $F_{2}^{c \bar{c}}\left(x, Q^{2}\right) \sim 0.4 A_{0}\left(Q^{2}\right) x^{-\epsilon_{0}}$ at small $x$, and the lines in Fig. 1, while calculated by NLO perturbative QCD, correspond to this to a very good approximation. As we have said above, the gluon density must also behave as $x^{-\epsilon_{0}}$ at small $x$.

In order to apply DGLAP evolution, according to (1) we need $^{1}$ the elements of the evolution matrix (2c) at $N=0.4$. We find that, for values of $N$ close to this, good fits are

$$
\begin{aligned}
& \mathbf{P}^{0}(N)=\left[\begin{array}{ll}
-0.0352299-2.41822 N+0.743989 N^{2} & 2.58893-2.2144 N+1.08471 N^{2} \\
18.4131-49.7311 N+42.476 N^{2} & 39.0374-115.451 N+96.1001 N^{2}
\end{array}\right], \\
& \mathbf{P}^{1}(N)=\left[\begin{array}{ll}
-1111.55-3187.40 N+-2685.19 N^{2} & -943.22+2952.70 N-2460.29 N^{2} \\
-636.76+2070.57 N-1707.29 N^{2} & -114.61+383.00 N-359.98 N^{2}
\end{array}\right] .
\end{aligned}
$$

of $(1-x)$ given by the dimensional counting rule [21], that is, $(1-x)^{7}$ for each of the two pomeron terms, and $(1-x)^{3}$ for the reggeon term. Although this is not strictly correct, it is better than not doing it, at least if $Q^{2}$ is not too large. Its effect is small for small $x$, though not completely negligible, and it makes the fit to the $x<0.001$ data valid some way beyond that range and therefore to larger $Q^{2}$ : see Fig. 5. The fits in this figure have

$X_{0}=0.03527, \quad X_{1}=0.03786, \quad X 2=0.06445$,

$Q_{0}^{2}=7.03262, \quad Q_{1}^{2}=1.68993 \times 10^{-5}, \quad Q_{2}^{2}=0.00192$.

The $\chi^{2}$ per data point is 0.95 for all the data with $Q^{2}$ between 3.5 and $45 \mathrm{GeV}^{2}$ and $x<0.001$.

\section{Perturbative QCD evolution}

We work to next-to-leading order in $\overline{M S}$ perturbative QCD, with [22]

$\alpha_{S}\left(M_{Z}^{2}\right)=0.1183$.

We follow the standard procedure [15] of changing from 4 to 5 flavours at $Q^{2}=m_{b 0}^{2}$ and making $\alpha_{S}\left(Q^{2}\right)$ continuous there, resulting in

$\Lambda_{5}=230 \mathrm{MeV}, \quad \Lambda_{4}=328 \mathrm{MeV}$.

For the range of $Q^{2}$ values that will concern us the contribution to $F_{2}\left(x, Q^{2}\right)$ from $b$ quarks is [23-25] negligibly small. Pomerons couple equally to $u$ and $d$ quarks. In the case of the soft pomeron, we know $[10,11]$ that the coupling to $s$ quarks is rather weaker than to $u$ and $d$ and, as may be deduced from the data in Fig. 2, very much so to $c$. However, the data for $F_{2}^{c \bar{c}}\left(x, Q^{2}\right)$ indicate that, for reasons that are not understood and to a very good approximation, the hard pomeron couples equally to all four quarks. To verify this, note that if this is
We choose some value $\bar{Q}^{2}$ of $Q^{2}$ and calculate $\Sigma\left(\bar{Q}^{2}\right)$ and its derivative $\Sigma^{\prime}\left(\bar{Q}^{2}\right)$ using the form of $A_{0}\left(Q^{2}\right)$ we obtained from the fit (6) to $F_{2}\left(Q^{2}\right)$. The DGLAP Eq. (2b) with $N=0.4$ then determines $G\left(\bar{Q}^{2}\right)$. This provides the input to the evolution in both directions from $Q^{2}=\bar{Q}^{2}$. Figure 6 shows the percentage difference for the choice $\bar{Q}^{2}=9 \mathrm{GeV}^{2}$. The plot in this figure is quite sensitive to the choice of $\bar{Q}^{2}$. It is well known that perturbative evolution is valid only at sufficiently large $Q^{2}$; we conclude that this means greater than about $5 \mathrm{GeV}^{2}$. Figure 7 shows how $G\left(Q^{2}\right)$ and $\Sigma\left(Q^{2}\right)$ evolve with $Q^{2}$. The value $\bar{Q}^{2}=9 \mathrm{GeV}^{2}$ gives agreement with the gluon density at very large $Q^{2}$ obtained from the conventional approach to perturbative evolution [26]: see Fig. 8. At these values of $Q^{2}$ the effective power $(1 / x)^{\epsilon}$ for the behaviour of $x g\left(x, Q^{2}\right)$ has become so large that the conventional approach is now valid.

We observe that the small values of $x$ that we consider in this paper contribute very little to the momentum sum rule. It is not trivial to achieve momentum conservation [27-29] but this is done by suitable choice of the parton densities at larger values of $x$.

\section{Applications}

We now apply the gluon density (1) shown in Fig. 7 from the NLO calculation of $G\left(Q^{2}\right)$ using the perturbative evolution formula (1b). A good fit in the range $5<Q^{2}<200 \mathrm{GeV}^{2}$ is

$G\left(Q^{2}\right)=X_{g}\left(Q^{2} /\left(Q_{g}^{2}+Q^{2}\right)\right)^{\eta_{1}}\left(1+Q^{2} / Q_{g}^{2}\right)^{\eta_{2}}$,

\footnotetext{
1 We are grateful to the authors of reference [15] for making available to us the numerical values of the elements of $\mathbf{P}\left(N, \alpha_{s}\left(Q^{2}\right)\right)$.
} 


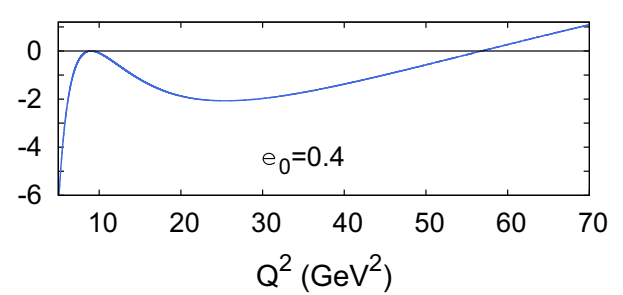

Fig. 6 Percentage difference between the evolved $\Sigma\left(Q^{2}\right)$ and that obtained from the fit to $A_{0}\left(Q^{2}\right)$ from the small- $x$ data for $F_{2}\left(x, Q^{2}\right)$

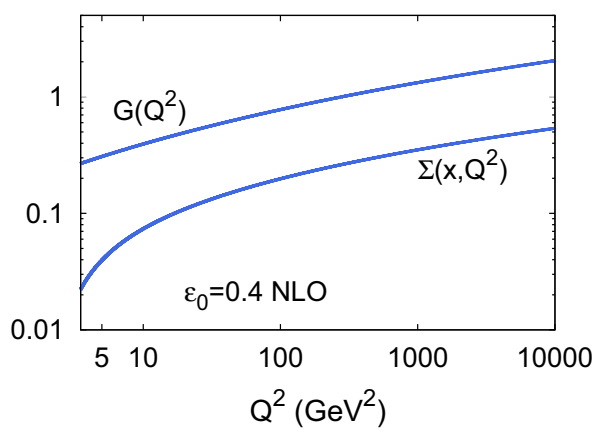

Fig. 7 Evolution of $G\left(Q^{2}\right)$ and $\Sigma\left(Q^{2}\right)$

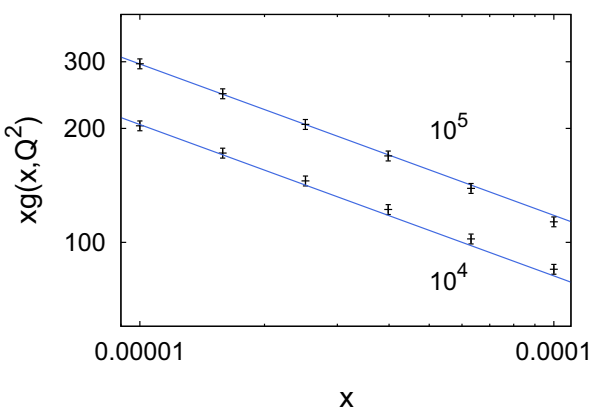

Fig. 8 Evolved gluon density at $Q^{2}=10^{4}$ and $10^{5} \mathrm{GeV}^{2}$. The points are the results [26] of conventional evolution according to HERAPDF20

with

$X_{g}=0.4338, \quad \eta_{1}=0.437, \quad \eta_{2}=0.252 \quad Q_{g}^{2}=9.128$.

Figure 1a shows the NLO calculation of $F_{2}^{c \bar{c}}\left(x, Q^{2}\right)$ using an adaptation of a program ${ }^{2}$ originally written by Laenen, Riemersma, Smith and van Neerven [30]. We use the NLO running mass $m_{c}\left(Q^{2}\right)$ with [22],

$m_{c}\left(m_{c 0}\right)=m_{c 0} \quad m_{c 0}=1.27 \mathrm{GeV}$.

A good fit, for $5<Q^{2}<10^{4} \mathrm{GeV}^{2}$, is provided by

$\left(m_{c}\left(Q^{2}\right)\right)^{2}=m_{c 0}^{2} /\left(1+0.3438 \log \left(Q^{2} / m_{c 0}^{2}\right)\right)$.

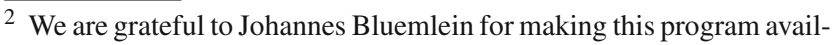
able to us.
In both $\alpha_{S}\left(Q^{2}\right)$ and $x g\left(x, Q^{2}\right)$ we replace $Q^{2}$ with

$\mu^{2}=Q^{2}+4\left(m_{c}\left(Q^{2}\right)\right)^{2}$.

Our calculation, whose results are shown in Fig. 1, includes only the gluon-induced charm production. The cross section for charm electroproduction is the sum of two terms, $e g \rightarrow$ charm and $e q \rightarrow$ charm, each convoluted with the corresponding parton density. Figure 1 shows that the first alone, calculated up to order $\alpha_{s}^{2}$, fits the data well. For the second, the order $\alpha_{s}^{2}$ term is the first term in the perturbative expansion. It turns out to be small at small $Q^{2}$, but it is negative because of the inclusion of a counterterm to cancel a collinear divergence, ${ }^{3}$ and therefore it is a very poor approximation to the exact expression. So it is appropriate to neglect it.

We perform an exactly similar calculation of $F_{2}^{b \bar{b}}\left(x, Q^{2}\right)$, with [22]

$m_{b}\left(m_{b 0}\right)=m_{b 0} \quad m_{b 0}=4.18 \mathrm{GeV}$

and the fit

$\left(m_{b}\left(Q^{2}\right)\right)^{2}=m_{b 0}^{2} /\left(1+0.179 \log \left(Q^{2} / m_{b 0}^{2}\right)\right.$.

The resulting calculations are shown in Fig. 9, together with the data [23-25].

We have said that, to calculate the $F_{L}\left(x, Q^{2}\right)$, it is sufficient to use the LO formula [31]

$F_{L}\left(x, Q^{2}\right)=K\left(x, Q^{2}\right)+\frac{4 \alpha_{s}\left(Q^{2}\right)}{3 \pi} \int_{x}^{1} \frac{\mathrm{d} y}{y}\left(\frac{x}{y}\right)^{2} F_{2}\left(y, Q^{2}\right)$

where the contribution of the charm quark to $K\left(x, Q^{2}\right)$ is

$$
\begin{aligned}
K^{c}\left(x, Q^{2}\right)= & 2 e_{c}^{2} \frac{\alpha_{s}\left(\mu^{2}\right)}{\pi} \int_{x \mu^{2} / Q^{2}}^{1} \mathrm{~d} y\left(\frac{x}{y}\right)^{2} \\
& \left.\times\left[\left(1-\frac{x}{y}\right) v-\frac{2 m_{c}^{2}\left(Q^{2}\right) x}{Q^{2} y} L\right)\right] g\left(y, \mu^{2}\right)
\end{aligned}
$$

with $\mu^{2}$ defined in (13) and

$v^{2}\left(Q^{2}\right)=1-\frac{4 m_{c}^{2}\left(Q^{2}\right)}{Q^{2}(y / x-1)}, \quad L=\log \left(\frac{1+v}{1-v}\right)$.

The light quarks contribute similarly, with zero mass. The calculations are compared with data $[16,32]$ for $F_{L}\left(x, Q^{2}\right)$ in Fig. 4. The circles in Fig. 10 show the calculated points for the reduced cross section (4), together with the 2015 joint HERA data [20]. The lines in this figure are the fits to $F_{2}\left(x, Q^{2}\right)$ from the 2010 HERA data [19]. The $\chi^{2}$ per data point is 0.96. Although, according to Fig. 6, the DGLAP evolution is not valid below $Q^{2}=5 \mathrm{GeV}^{2}$, the evolved gluon density gives good results for $F_{L}\left(x, Q^{2}\right)$ down to $3.5 \mathrm{GeV}^{2}$.

\footnotetext{
${ }^{3}$ We are grateful to Eric Laenen for explaining this to us.
} 
Fig. 9 NLO calculations of $F_{2}^{b \bar{b}}\left(x, Q^{2}\right)$ with data [23-25] from ZEUS and $\mathrm{H} 1$ at various values of $Q^{2}$

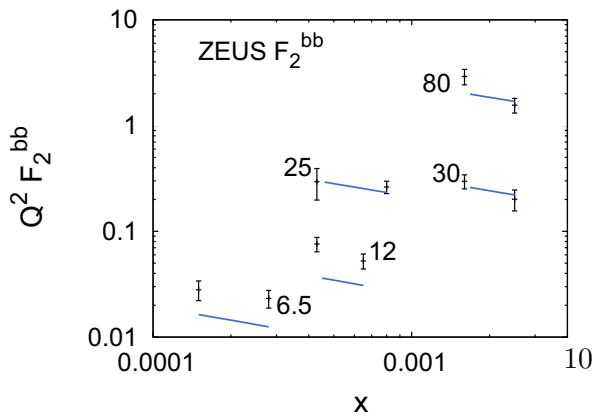

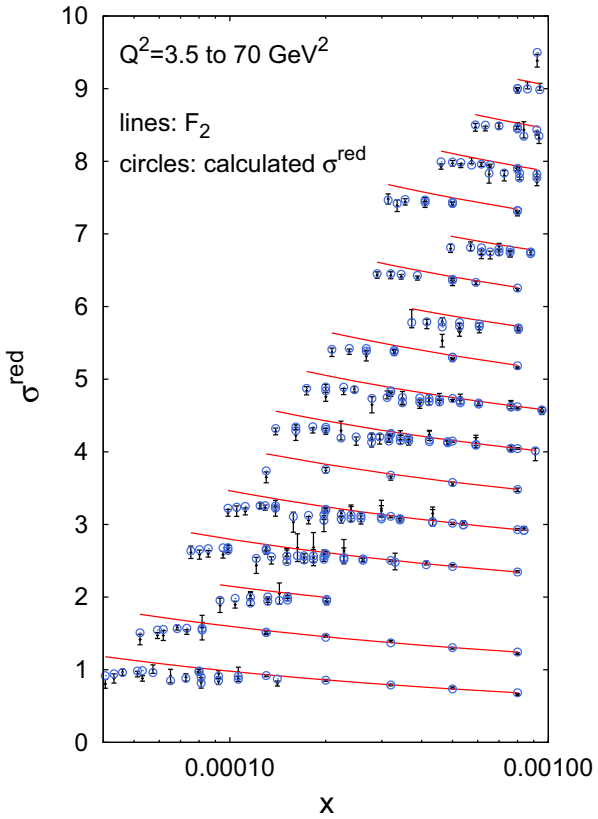

Fig. 10 Data [20] for the reduced cross section. The circles denote the calculations and the lines the fit to $F_{2}\left(x, Q^{2}\right)$ from the 2010 data [19] -0.25 is added to the data points for each successive value of $Q^{2}$

Acknowledgements We thank Marco Guzzi for helpful discussions on DGLAP and the CT14 analysis.

Open Access This article is distributed under the terms of the Creative Commons Attribution 4.0 International License (http://creativecomm ons.org/licenses/by/4.0/), which permits unrestricted use, distribution, and reproduction in any medium, provided you give appropriate credit to the original author(s) and the source, provide a link to the Creative Commons license, and indicate if changes were made. Funded by SCOAP ${ }^{3}$.

\section{References}

1. S. Catani, F. Hautmann, Nucl. Phys. B 427, 475 (1994). arXiv:hep-ph/9405388

2. J.R. Cudell, A. Donnachie, P.V. Landshoff, Phys. Lett. B 448, 281 (1999)

3. J.R. Cudell, A. Donnachie, P.V. Landshoff, Phys. Lett. B 526, 413 (2002)
4. G. Altarelli, R.D. Ball, S. Forte, Nucl. Phys. B 599, 383 (2001)

5. A.A. Almasy, N.A. Lo Presti, A. Vogt, JHEP 01, 028 (2016). arXiv: 1511.08612

6. A. Donnachie, H.G. Dosch, P.V. Landshoff, O. Nachtmann, Pomeron Physics and QCD (Cambridge University Press, Cambridge, 2002)

7. A. Donnachie, P.V. Landshoff, Phys. Lett. B 533, 277 (2002). arXiv:hep-ph/0111427

8. H1 and ZEUS collaborations, H. Abramowicz et al., Eur. Phys. J. C 73, 2311 (2013). arXiv:1211.1182. https://www.desy.de/h1zeus/ combined_results/index.php

9. A. Buckley et al., Eur. Phys. J. C 75, 132 (2015). arXiv:1412.7420. https://www.hepforge.org/archive/lhapdf/pdfsets/6.1/

10. A. Donnachie, P.V. Landshoff, Nucl. Phys. B 231, 189 (1984)

11. A. Donnachie, P.V. Landshoff, Phys. Lett. B 296, 227 (1992)

12. J.R. Cudell, K. Kang, S.K. Kim, Phys. Lett. B 395, 311 (1997)

13. A. Donnachie, P.V. Landshoff, Phys. Lett. B 727, 500 (2013). arXiv: 1309.1292

14. A. Donnachie, P.V. Landshoff, Phys. Lett. B 437, 408 (1998). arXiv:hep-ph/9806344

15. R.K. Ellis, W.J. Stirling, B.R. Webber, $Q C D$ and Collider Physics (Cambridge University Press, Cambridge, 1996)

16. Z.E.U.S. Collaboration, S. Chekanov et al., Phys. Lett. B 682, 8 (2009). arXiv:0904.1092

17. H1 collaboration, F.D. Aaron et al. Phys. Lett. B 393, 452 (1997). arXiv: 1012.4355

18. S. Moch, J.A.M. Vermaseren, A. Vogt, Phys. Lett. B 606, 123 (2005)

19. H1 and ZEUS collaborations, F.D. Aaron et al., JHEP 01, 109 (2010)

20. H1 and ZEUS collaborations, H. Abramovicz et al., arXiv: 1506.06042

21. R.G. Roberts, The Structure of the Proton (Cambridge University Press, Cambridge, 1990)

22. Particle Data Group, http://pdg.lbl.gov

23. ZEUS collaboration, H. Abramowicz et al., JHEP 09, 127 (2014). arXiv: 1405.6915

24. H1 collaboration, A. Aktas et al., Eur. Phys. J. C 40, 349 (2005). arXiv:hep-ex/0411046

25. F.D. Aaron et al., Eur. Phys. J. C 65, 89 (2010). arXiv:0907.2643

26. https://www.hepforge.org/archive/lhapdf/pdfsets/6.1/

27. S. Catani et al., Nucl. Phys. B Proc Suppl 29 A, 182 (1992)

28. R.K. Ellis et al., Phys. Lett. B 348, 582 (1995)

29. F. Hautmann, arXiv:hep-ph/9506303

30. E. Laenen, S. Riemersma, J. Smith, W.L. van Neerven, Nucl. Phys. B 392, 162 (1993)

31. V.M. Budnev et al., Phys. Rep. C 15, 181 (1974)

32. H1 collaboration, F.D. Aaron et al. Eur. Phys. J. C 71, 1579 (2011). arXiv: 1012.4355 